\title{
Alzheimer's Prevention Initiative
}

\author{
Gabrielle Strobel \\ Alzheimer Research Forum
}

\section{PART 1: VISION OF SHARED PREVENTION TRIALS LURES PHARMA TO TABLE}

On 26 January 2010, at an ordinary airport hotel in Phoenix, Arizona, an extraordinary gathering unfolded. High-level representatives of 19 different pharma, biotech, and medical companies from across the U.S. and Europe - businesses that compete fiercely for the same market - spent the entire day cooped up in one room. There they engaged in a searching, at times surprisingly candid, discussion with academic research leaders, funders, and regulatory and statistics experts. Their topic? How they could set aside their competition in order to advance a shared vision of testing candidate drugs in people who are at imminent risk for Alzheimer's disease (AD) but have no symptoms. In other words, these are future patients who, pathologically speaking, may already have AD but as yet without the dementia that epitomizes the disease in most people's minds.

AD prevention has become a favorite buzzword of late, but approaching this goal with clinical trials of candidate AD drugs is difficult to do. This meeting represented perhaps the most advanced effort to date to actually pull it off. Called the Alzheimer's Prevention Initiative (API), the effort is the brainchild of Eric Reiman and Pierre Tariot at the Banner Alzheimer's Institute and the Arizona Alzheimer's Consortium in Phoenix, Arizona. They and their Banner colleagues had invited the group of 50 people to this meeting. Riding the collaborative wave created by previous shared efforts such as the Alzheimer's Disease Neuroimaging Initiative (ADNI), the Alzheimer's Disease Cooperative Study (ADCS), and the Dominantly Inherited Alzheimer Network (DIAN), these scientists are trying to build a coalition of academic and industry leaders with other stakeholders to begin, already in 2011 , biomarker-driven drug trials initially in two separate sets of people who, based on their genetic status and age, are at the highest imminent risk of symptomatic AD. One group is middle-aged adult relatives of a large extended family near Medellin, Colombia, who are stricken with a deterministic early-onset presenilin 1 mutation. The other group are people in their sixties and seventies in the U.S., and potentially abroad, who carry two copies of the ApoE4 gene. The API has joined forces with DIAN, the international network for families with dominantly inherited (i.e., A $\beta$ PP- and PS-mutant) AD, which is already enrolling for an observational study and has begun planning its own drug study to offer to DIAN participants in the next year or two, as well.

"My dream is to get parallel preclinical trials going in these high-risk people and also the general population. We want to launch an era of prevention research where the field at large evaluates pre-symptomatic treatments rigorously and rapidly in randomized clinical trials," said Reiman. If this seems ambitious, that is because it is. Talking about the benefits of prevention of AD is stating the obvious, but the day's discussions highlighted the fact that many hurdles remain. They also made clear that pharma's pre-competitive spirit comes to a cold stop when the discussion broaches their own or their competitors' drugs. Even so, most attendees urged Reiman and Tariot to press on with their proposal and indicated that they would want to be part of it.

That is in part because concern is growing in academia and industry that the conventional mode of testing drug candidates in people with mild to moderate AD using parallel-arm study designs may not produce impressively effective therapies any time soon. There is a palpable concern that this conventional treatment trial design could sink the prospects of what might have been a respectable pre-symptomatic therapy before a way is 
found to test for the latter. A new model is needed, and in Phoenix, leading scientists of all backgrounds voiced remarkable consensus that the time is right to break out of the mold. Moreover, it appeared the company scientists, who work within the strategic and legal constraints of big pharma, mostly removed from patients, welcomed initiatives from physician-researchers who personally take care of people they know will get AD fairly soon and who are chomping at the bit to try to stave off that day. "In our blood-and-guts, clinical, dayto-day environment, we come under a lot of pressure to act boldly. That is a good thing," said Tariot.

To the general public and to many doctors, Alzheimer disease is largely synonymous with dementia and the progressive loss of self and independence. Typically, people develop symptoms, seek a diagnosis, and then take drugs. However, since the advent of biomarker and imaging research as far back as the early 1990s, scientists have gradually been parsing what it means to have AD with greater nuance. They have observed the disease's molecular changes start to take place many years before people realize anything might be amiss in their brains. With this, the notion of a pre-symptomatic, biologically defined decade or so began to take shape. By this notion, the period of overt suffering most people equate with having $\mathrm{AD}$ would constitute merely the denouement of a much longer disease process. As is the case in other medical conditions, the end stage of organ failure of a disease process may be hardest to treat, raising hopes for a wave of drug trials in this newly defined, clinically "silent" phase. An oft-quoted analogy is heart disease, where preventing atherosclerosis is more successful than treating heart failure. It also requires different drugs. The deeper implication here is that some of the investigational drugs currently tested for treatment might do better in prevention, and that a whole separate set of drugs will be needed for treatment.

Scientifically speaking, the first trials proposed by API would represent pre-symptomatic treatment, giving study medication to people who are cognitively normal. It would be called secondary prevention if there is evidence of the brain changes that signal predisposition to clinical AD. Scientists use various terms and definitions to best describe the disease stage prior to mild cognitive impairment or prodromal AD. However, practically speaking from the perspective of the "patients," the success of such trials would amount to prevention, or at least to a reprieve before their minds start to fail them.

Newly formulated by Reiman and Tariot, the vision of drug trials for pre-symptomatic AD was previously considered too difficult to realize. "This was a pipe dream not long ago," Tariot said. Now, its time may have come, industry and academic leaders agreed in Phoenix. To a reporter who has covered the issue before, the change in tone was striking. As recently as two years ago, when industry scientists were asked about testing their most attractive candidate therapy in prevention trials, a positive reply would go along the lines of: "We would like to do this but...", and then a conversation about problem after problem would ensue. More typically, pharma scientists would simply point out that their senior management would reject such trials because it viewed the risks to the candidate drug and the company's investment in it as exceeding the potential benefit. Last month, however, AD program leaders from company after company greeted the API proposal positively. This article will not attribute quotes by industry scientists to protect open discussion at forums such as the one in Phoenix and to avoid the need for lengthy internal review by corporate communications. Here are unattributed samples of what they said about the proposed initiative:

"Fantastic."

"Prevention is where we have to go."

"The time is right."

"I am very encouraged - for years we have wanted to do this kind of study in genetic populations."

"This is the right thing. That it is difficult is just part of it, and we will try to work that through."

What has changed? Several trends are coming together. For one thing, imaging and biomarker data generated by ADNI as well as by observational studies in Reiman's and other centers worldwide have converged to a degree of consensus; they support planning presymptomatic trials that rely on these biomarkers for enrollment and/or for measuring success. Various amyloid imaging agents are in Phase III studies and might receive the Food and Drug Administration's blessing before long. A worldwide Quality Control program is gearing up to make $\mathrm{CSF} \mathrm{A} \beta /$ tau testing more robust. The Critical Path Institute, whose director, Louis Kirby, attended the Phoenix meeting, is working to speed up the FDA's formal acceptance of biomarkers. AD scientists are already detecting a subtle opening on the part of FDA regulators, who are increasingly accepting the notion that the presence of AD pathology in a person's brain prior to symptoms represents a disease state. An accelerated approval pathway based in large part on biomarker evidence exists and could potentially serve to guide the API. 
At the same time, the conventional model of drug testing is being called into question as anti-amyloid and other investigational therapies have so far performed below expectations. "There is a growing concern that these may be too little too late to exert a profound clinical effect once patients have even mild symptoms. We need to move earlier," said Reiman, and this stance now draws widespread support. In Phoenix, one company scientist recounted how the trial results of anti-amyloid drugs played internally at his company: "The weak trial results hit the hypothesis hard. In 2009, we were asked to present to senior management on the state of the amyloid hypothesis, and the outcome of that was that the best test of the hypothesis were prevention trials both in PS1 and in ApoE4 high-risk groups." This resonates throughout industry as scientists are preparing internally for what to do next once their present crop of experimental treatments has completed the current round of clinical trials.

This is not to say that researchers are giving up on the effort to treat symptomatic patients; large trials are continuing and hope is still high that some investigational medicines will show an effect alone or in combination. But at the same time, there is a growing sense among scientists that many of the currently known treatments may be most beneficial early in the process.

Specifically, scientists in Phoenix noted that the field is undergoing a paradigm shift whereby treatment of mild to moderate $\mathrm{AD}$ might require other classes of treatment (read: non-amyloid ones, because by then the disease has become independent of this pathology) than treatment of the preclinical period, where anti-amyloid investigational therapies seem more closely linked to the disease process. "We are in the middle of a sea change in AD realizing that," one pharma representative said.

Finally, public awareness of AD is growing, along with a sense that research participants at high risk for AD deserve access to the most promising drug candidates.

Despite the main news at this juncture - unison advice by industry to press ahead - readers should not expect to sign up for a prevention trial just yet. There are still plenty of obstacles to clear. The list includes drug safety, dose determination, trial design, genotype disclosure, among others. The 26 January 2010 meeting in Phoenix followed an earlier gathering of academic scientists, representatives of federal and private funders, and related groups that Reiman and Tariot had called in October 2009. There, too, the motto of the day was not to let challenges deter the overall initiative. "The issues have reached a stage of clarity where we must move forward even without complete consensus on how to solve these big remaining problems," concluded Paul Aisen of the University of California, San Diego, who directs the ADCS. Things are moving. Reiman and Tariot flew to Colombia earlier this month, exploring the installation of a cyclotron there to make PET compounds for amyloid imaging, meeting scientific staff and family members, and generally laying groundwork for the screening of the initial 1,000 prospective study participants.

\section{PART 2: CAN ALZHEIMER'S PREVENTION INITIATIVE BREAK A CATCH-22?}

Reiman was not alone in thinking about such trials applied to people at high risk for many years. The idea had been around ever since AD genetics had its first successes. Some physician-researchers who have cared for and studied genetic families have long dreamed about trying to protect the adult children and younger siblings of their patients from this disease with drug trials, as indeed did scientists who run observational studies of cognitively normal people carrying two copies of the risk gene ApoE4. As Reiman recalls it, what jolted him into action was a particular encounter with a PS1 mutation carrier who had sought to know his genetic status years before from another scientist. A proactive, no-nonsense, can-do businessman, this man had been participating in multiple observational studies by Reiman's and other research groups ever since finding out his genotype, all the while insisting that the scientists show him his data. One day in 2007, he walked into Reiman's office, slammed onto his desk PIB-PET scans of his brain that Bill Klunk of the University of Pittsburgh had run, and said, simply "Look. Here is my disease. Enough with just observation. When will someone try to find a prevention therapy for it?" "Speak of the urgent need!" Reiman told this reporter. He pledged to find a way to bring the makers of the most promising disease-modifying treatments to the table and gather the evidence needed to conduct prevention trials as soon as possible in people at the highest imminent risk of symptomatic AD. "I think of our conversation, this gentleman, and others like him every single day," Reiman said.

Reiman then drafted a proposal, and he and Tariot spoke privately with colleagues in science and related stakeholder communities such as the Alzheimer's Study Group to field advice, invite vetting of ideas, 
and build support. Converting the long-held ideal of prevention trials in high-risk folks into an action plan required an intense internal effort by a team of people at Banner. "The process was lengthy and laborious even before anything was made public," Tariot recalls. Along the way, the scientists fine-tuned the API to combine a general call to arms with two specific drug trials they hope to get going as early as next year. Now, Reiman, Tariot, and Jessica Langbaum of Banner are presenting the API publicly with an article in Biomarkers in Medicine [1]. Here is a summary.

At present, the scientists argue, the greatest roadblock in AD research is not the discovery of new candidate treatments, but the absence of effective means to evaluate those treatments rigorously and rapidly. Conventional prevention trial paradigms do not work for that. They require too many cognitively normal people (thousands in a trial), too many years (longer than a treatment's patent protection), and too many dollars for the whole field to evaluate more than one or two treatments at a time in this way. In fact, recent history of $\mathrm{AD}$ prevention research has featured but three large prevention trials, the ADAPT trial of two NSAIDs [2], the Gingko Evaluation of Memory Study [3] and the Women's Health Initiative Memory study of hormone replacement therapy [4,5]. What is needed are nimbler trials so more treatments can be evaluated more quickly.

This need invokes imaging and biochemical markers, but these are caught in a strange Catch-22, the authors argue. Observational studies such as the ADNI and others are providing a strong scientific rationale for biomarker use in pre-symptomatic trials, but that is not enough. Regulatory agencies at present are reluctant to approve pre-symptomatic treatments based on biomarker endpoints alone; rather, they expect sponsors to show that drug-induced changes in such markers reasonably predict that a person will benefit clinically, i.e., that the biomarker change truly helps. This evidence can come from separate randomized clinical trials, and it can be pieced together from across different trials of different treatments. Such biomarkersin-trials type of evidence is needed to establish which markers budge in response to which treatment, whether they change in the expected or in the opposite direction, and which biomarker changes portend a meaningful benefit. Alas, not nearly enough of this evidence is being generated these days because many drug sponsors hesitate to shoulder the added cost of assessing $\mathrm{AD}$ imaging and biomarkers in clinical trials of a given drug. Conventionally, such clinical trials pro- grams focus narrowly on advancing the drug at hand, not on gathering broadly relevant surrogate marker data along the way. That none of the biomarkers in question are by themselves FDA-approved (or "qualified," in FDA lingo) adds to the state of limbo. The upshot of it all is that drug sponsors are passing on costly presymptomatic trials while they see neither a clear path to approval nor a financial incentive. "This dilemma may at first seem...[insurmountable], leading to a sense of nihilism," the scientists write.

But inaction is not an option, they continue. Here are some reasons for why biomarker-driven presymptomatic trials should break the deadlock now.

- Recent projections predict 65 million AD cases worldwide by 2030 , even more in the early prediagnosis stages.

- Promising interventions spanning lifestyle, dietary factors, and approved medications exist; a good way to test them does not.

- Investigational treatments (anti-amyloid, anti-tau, others) are gathering safety and efficacy data in randomized clinical trials - enough, perhaps, to test them in prevention.

- Some of these investigational treatments may perform better in the disease's pre-symptomatic stages, especially those whose hypothesis is tied to the earliest-moving biomarkers.

- People at the highest risk for AD offer a stepping stone into broader prevention studies. Their risk could be genetic or evidenced by abnormal biomarker profiles.

- Imaging and CSF biomarkers to detect and track the brain changes of pre-symptomatic $\mathrm{AD}$ are in hand.

Regarding this last point, ADNI results and attendant power calculations support future trials. ADNI is not the only source of such data. Reiman and colleagues point out a 14-year-old, ongoing study in Phoenix that does essentially the same thing, sans CSF biomarkers, for cognitively normal, middle-aged people who carry either zero, one, or two copies of the AD risk allele ApoE4. Banner scientists and their colleagues at the Mayo Clinic Arizona have measured declining synaptic activity, shrinkage of the brain, and amyloid deposition in people with two copies of ApoE4, whose lifetime risk of developing AD is $91 \%$. This observational study and others like it have generated data that support the design of trials in which these imaging tools would evaluate an investigational treatment. In some cases, this could be done directly against a particular person's 
documented history of biomarker change. By now, a range of studies have documented abnormal changes in brain activity, volume, or CSF levels of $\mathrm{A} / \mathrm{t}$ tau in ApoE4 carriers, PS or A $\beta$ PP mutation carriers, or even in people without a defined genetic burden. Together, this body of evidence makes a strong case for using those same markers to evaluate treatments in people whose markers are changing, according to the Banner authors.

Here is how Reiman and colleagues intend to break the deadlock. In general, they define pre-symptomatic $\mathrm{AD}$ treatment as one that slows or stops an existing disease process and delays or prevents its symptoms. This shifts the balance of competing risks - those of impending clinical onset versus harm from an experimental medication with limited safety data - toward treatment in the eyes of trial participants, physicians, and regulatory agencies. The API proposal does not focus on primary prevention in the general population.

Specifically, the authors propose a new scientific paradigm and some public policy reforms. The science piece includes a series of preclinical treatment trials in defined groups of people (see Part 3 of this series), a call on all drug sponsors to embed into their ongoing and new drug trials measurement of the imaging and biomarkers that are performing well in ADNI and other longitudinal studies, and the building of national registries of cognitively normal people who express interest in participating in pre-symptomatic AD trials. The API's policy piece includes some ideas previously formulated in the report of the Alzheimer's Study Group [6]. For example, resetting the clock for patent protection to the time of completion of lengthy prevention trials might galvanize industry interest in such trials. Extending market exclusivity has proven successful for orphan drugs, the authors note, and could be tried as a financial incentive for AD prevention, too. Other ideas include federal funding for prevention trials of off-patent drugs and for inclusion of biomarker assessments into proprietary drug trials in return for full publication of the biomarker results.

\section{PART 3: TRIALS IN COLOMBIA AND THE U.S. FOR THOSE AT HIGHEST RISK?}

During two recent all-day discussions with academic, funding, and industry leaders, and now in a published article, Eric Reiman, Pierre Tariot, and Jessica Langbaum of the Banner Alzheimer's Institute in Phoenix, Arizona, have laid out a vision for pre- symptomatic treatment trials that emphasize use of biomarkers to evaluate investigational drugs in people who find themselves in a particularly precarious situation.

One such group is middle-aged carriers of the E280A PS1 mutation discovered by Francisco Lopera in the state of Antioquia in central/northwestern Colombia. Together with Kenneth Kosik at the University of California, Santa Barbara, who co-identified this mutation in 1997 [7], Reiman, Tariot, and Lopera are jointly preparing for this trial. Lopera and numerous collaborators in U.S. laboratories have analyzed this form of AD for 20 years, demonstrating plaque deposition [8]), abnormalities in cerebral perfusion [9], modification by ApoE [10] and describing its early onset [11]. Lopera, a neurologist, and his colleagues have followed many relatives in this kindred for several decades.

The E280A mutation affects the largest group of people worldwide of the more than 200 presenilin mutations known to date. Size matters, because the extreme rarity of autosomal-dominant $\mathrm{AD}$ severely limits the power of clinical research and indeed is one reason why the DIAN has banded together across three continents. Living members of the E280A family tree number about 5,000 individuals spread among 25 families who live in the historically isolated region of Antioquia, Colombia. Nearly half of this "family" live in the city of Medellin, the capitol of Antioquia. Some 1,000 of those are estimated, and 400 already known, to carry the mutation. More than 100 currently known carriers are cognitively normal and older than 40 , i.e., candidates for a pre-symptomatic trial now, according to Reiman. Some 2,000 family members have been genotyped, and more may choose to obtain genetic testing once a treatment trial is on offer.

Carriers start noticing subtle memory problems from age 40 on, Lopera said. Their median age of onset for MCI is 45 years and 47 years for AD dementia. Certain cognitive tests, such as the Buschke memory test and tests measuring intrusion errors, can flag those who are likely to develop dementia 10 years before they do, Lopera said at an API planning meeting held October 2009 in Phoenix.

To take place successfully in Colombia, the proposed trial and attendant observational research will need to clear added cultural, financial, and logistic hurdles, as many trial participants are poor and have but a grade school-level education. The effort requires political sensitivity and compassionate support of the study population with appropriate community services, Reiman emphasized. But it is nonetheless doable, 
Lopera assured attendees at the October 2009 meeting in Phoenix. "The families in Colombia are waiting for us to find the solution," Lopera said. At the same meeting, Kosik confirmed, "These people are very keen to participate in clinical trials. They know they are likely to get this disease."

They know from witnessing the disease in their families, not based on their carrier status. Because genetic counseling is unavailable in Colombia, Lopera does not disclose the PS1 test result. For the trial, too, participants would not learn their genotype. To balance the scientific need for randomization and placebo control with the ethical responsibility to protect study participants, the API scientists intend to treat only carriers with study drug, no non-carriers, and to enroll noncarriers into the control group so the study can be blinded. For deeper insight into the discovery and cultural circumstances of this Andean kindred, see The Fortune Teller, Kosik's essay in The Sciences [12].

For this trial, Reiman and his colleagues have obtained initial funding to start screening some 1,000 relatives for inclusion. More funding is needed to conduct the trial and support participating local communities in consultation with Lopera and his team.

Because the Colombian families face essentially the same genetic form of $\mathrm{AD}$ as do participants in the ongoing DIAN, the API and DIAN have decided to join forces for what will be complementary presymptomatic AD trials in eFAD gene carriers. DIAN started out as - and is currently enrolling for - an NIAsupported observational study to characterize the presymptomatic phase of $\mathrm{AD}$ in cognitively normal carriers using a suite of biomarkers much like those in ADNI; DIAN plans for treatment studies based primarily on biomarker endpoints. In contrast, API is starting out as a clinical trials program; it aims to relate a treatment's biomarker effects to clinical outcomes. But both efforts share the vision of pre-symptomatic treatment trials that richly embed biomarkers, and there is much they could do together. For example, if both initiatives run drug trials, family members in one initiative who do not meet inclusion criteria for a given trial at the time they undergo screening may well qualify for a trial in the sister initiative, Reiman said. For its part, DI$\mathrm{AN}$ is exploring options to offer a clinical trial in about the same time frame as API. Coordinating with DIAN may, in turn, help Lopera and the API team to expand observational biomarker studies in Antioquia.

In addition, the API leaders have set their sights on a second group of people at exceptionally high risk of AD. They are those who carry two copies of the AD risk allele ApoE4 and have aged to near the median age of onset for this disease, 68 years. ApoE4 is a risk gene, not a deterministic one. Still, past the age of 60 years, the risk of developing AD in people with two ApoE4 genes grows so high that a secondary prevention trial becomes feasible, Reiman argued. Their relative risk of $\mathrm{AD}$ rises to 30:1 in community-based prospective research, and to 60-90\% in cohort or case-control studies. Scientists in Phoenix agreed that the absolute risk of $\mathrm{AD}$ in this age group remains to be better defined; however, a large enough percentage of ApoE4 homozygous people at that age develop AD symptoms within the next five years that it becomes statistically possible to detect a difference between treatment and placebo.

Moreover, prior observational imaging and biomarker studies have provided a body of evidence suggesting that the brains of many people with this combined genetic/age risk already show signature AD-like changes [13-17]. Their memory declines faster as they age, too [18]. In the ApoE API trial, also, imaging and biomarker measures would play a lead role, both to measure a drug effect and to tie drug-induced biomarker changes to later clinical protection. The details of how to accomplish this were the topic of intense discussion at an industry advisory meeting of the Banner scientists hosted in Phoenix last month; the trial's basic premise drew wide support.

Such trials in ApoE4-positive people would begin to answer the question in the minds of some scientists of whether autosomal-dominant $\mathrm{AD}$ and late-onset AD will respond to the same kinds of treatment. In essence, this trial would make it possible to start assessing whether results from the Antioquia and DIAN trial hold true more broadly for the vast majority of people with AD.

How would the scientists pull off this trial? The ApoE4/4 genotype occurs in some $3 \%$ of the U.S. population, and current power calculations based on prior observational research call for some 400 trial participants. Many people who are initially screened for a study fail one of its inclusion criteria and some change their minds about enrolling; therefore, the API researchers are aiming to recruit for screening some 20,000 to 50,000 people between the ages of 60 and 80 years. Prospective participants would undergo genotyping for ApoE; people who have already done so in the course of their cardiovascular care, or as users of personal genomics services, would be eligible as well. In order to reach this many people interested in participating in such a study, the API researchers are building a screening registry. They are also reaching out 
to other scientific groups who have registries or are already working with ApoE-genotyped volunteers for observational studies, from ADNI to ADCS to adult children studies, as well as epidemiological cohorts and REVEAL.

For this trial, the API researchers currently envision disclosing ApoE genotype to participants, but they intend to manage this process carefully in accordance with findings from the REVEAL studies of ApoE genotype disclosure. "This study found that people weather genotype information better than previous scientific consensus groups had expected," Reiman said in Phoenix. In general, concerns about ApoE genotype disclosure are seen to become less acute once a trial is on offer because it gives people a way to fight their risk beyond lifestyle changes, scientists in Phoenix agreed. One downside of disclosure the API scientists are weighing is that all participants' adult children would automatically know that they carry at least one copy of ApoE4, increasing their own lifetime risk, even if they did not seek that information. Avoiding disclosure is possible but would require including noncarriers, hence drive up cost. The API scientists are hoping that the National Institute on Aging will support their initiative and allow them to seek federal funding for it this year.

For both trials, the researchers initially suggested a five-year, double-blind, placebo-controlled design with clinical, cognitive, FDG and amyloid PET, structural MRI, and CSF measurement at various visits. The scientists would analyze data midway through the trial to see whether, as judged largely on the basis of the biomarker data, the treatment was likely doing any good. Then, they either would continue or declare the initial treatment futile and switch to a different one. This design was intensely debated (see Part 4 above) and is not etched in stone yet, Reiman said. Whichever final design details prevail, the key point about the overall initiative is that it could provide a foundation and much-needed impetus for additional pre-symptomatic, randomized controlled trials in the future, he noted. On this, attendees at both API planning meetings agreed.

Also for both trials, the API researchers stressed that they wish to conduct them as much as possible in a precompetitive fashion. A drive to seize common ground and work jointly on problems that hold back all otherwise competing pharma companies has buoyed ADNI and the ADCS. "The public-private collaboration on ADNI has worked so well that now we think we can do anything," said Paul Aisen of the University of California, San Diego, who heads ADCS and participates in
ADNI and DIAN. Representatives from different pharma companies as well as from the Foundation of the NIH agreed, and Reiman clearly wants to capitalize on the momentum. One way in which API foresees doing so is by tying joint funding and advice to open public access to the biomarker data these trials will generate. These data may speed the qualification of a surrogate marker, which in turn would boost the clinical development of AD treatments in general, not just the particular one(s) chosen for the API trials. This point, however, proved to be sticky. Once one competitor's drug is on the table, shared interests "get stuck in quicksand," as one pharma attendee phrased it. The day's discussion made clear that a bright line emerges where drugs are concerned, especially investigational ones, and carving out real meaning for the "one for all, all for one" spirit will take creative thinking and deft negotiation. But everyone professed to the cause, at least verbally thus far (see Part 4).

\section{PART 4: FOR SHARED PREVENTION TRIALS, DEVIL IS IN THE DETAILS}

In Phoenix, a roomful of senior industry and academic scientists, and regulatory and statistical advisers, dug deep into the details of a proposed pre-symptomatic trials initiative put forward by Eric Reiman, Pierre Tariot, and their colleagues at the Banner Alzheimer's Institute in Arizona's state capital. After near-unanimous praise for the initiative, the attendees delved into specifics and in the process exposed topics that need more preparation before any trial can actually start. Selecting a drug, setting the right dose, and designing the trial to greatest effect were among the hot button issues, as were questions about how to clear a regulatory path and persuade companies to hand over precious drugs to a public-private study they do not control. None of these issues appeared grave enough to derail the initiative; rather, they define the current cutting edge of how academia and industry forge new collaborations in order to move toward prevention research.

\section{Drug Selection: Can We Have Privacy, Please!}

If anyone was hoping that representatives from 19 companies would meet in one room, openly discuss the pros and cons of their investigational treatments, and vote on the best candidate for a public-private presymptomatic trial, those hopes fizzled quickly when the discussion neared the issue. Pharma scientists who had been discussing general issues about the API with remarkable candor, quickly clammed up. 
"I am not allowed to discuss [my company's] or any other drugs."

"Cannot have this conversation."

What is more, some academic leaders joined in, saying that their various advisory roles and confidentiality agreements with pharma companies precluded them, as well, from discussing investigational drugs in the assembled group. In essence, different people in the room knew different parts of the full truth about any given drug. Pharma people tend to know what is public, confidential to their own company, and purchased from competitive intelligence companies; some academics know only what is published and their own research; other academics may know what is published, their own research, plus confidential information from one or several companies, but not all companies. Taken together, none of these groups is in a position to talk fairly about drugs, and the last has a hard time intellectually managing their conflicts in this regard, Lon Schneider wrote to the Alzheimer Research Forum.

Things took an almost comical turn for a moment when a prospective trial leader insisted, "At some point, the rubber hits the road and we need to discuss the actual drugs. Do we have to exclude the world leaders on these drugs from the discussion?" Awkward silence. Next question: "Can we at least talk about what is publicly known about these drugs, or do all our discussions have to stay in the abstract?" On that, both industry and academics acknowledged that, in practice, they could not tell apart clearly enough what was public and what was not about a given treatment, therefore would not discuss them at all. "We cannot discuss individual drugs here without getting hopelessly mired in conflict of interest," said one pharma representative to nods around the room.

And so it was. The entire day passed without a specific treatment name having been uttered by any of the attendees. But the stalemate did not last long. It gave way to consensus that it is a question not of will but of process - and really not so difficult. In essence, an independent group of academic scientists can solicit advice from companies about what kind of information they need in order to choose the best candidate drug for a trial-deep safety, pharmacokinetic, pharmacodynamic data, performance on specific genetic backgrounds, etc. Then this group can invite companies confidentially to nominate a compound supported by this information. The academic group conducts its own due diligence on the nominations and makes its selection. This has been done before, and partial models, such as the ADCI or the TURNS process in schizophrenia research, can guide the API.

Another difficult question concerned how to preserve precompetitive space as the API proceeds. How to define shared gains once a drug has come into play will require more thought. "This is very different from ADNI," several scientists cautioned. The day saw some discussion about whether a drug company, which views an investigational treatment that has consumed millions of dollars in development cost as a precious "asset," would yield control of that asset to a public-private consortium. However, that this issue is shifting became clear when others pointed to prior industry-public agreements on how to share control, for example, those negotiated by ADCS, while some other pharma representatives assured the API scientists that "If you build it, we will come." A growing number of pharma scientists appeared to take the view that testing their drugs in earlier stages would not put an asset at unacceptable risk, but on the contrary, might in fact save an asset whose performance in clinically ill patients did not live up to expectations. All agreed that pharma scientists have more work to do to communicate these issues to senior management, where decisions about which trials to move forward are made.

Similar public-private arrangements have been negotiated in the past for proprietary drugs in the areas of estrogen replacement therapy, stroke, sickle cell anemia, and HIV prevention, though those arrangements involved primarily marketed drugs. Finally, several industry researchers suggested that positioning participation in API as a benevolent act toward a societal crisis could free industry from some of the usual proprietary restraints in releasing investigational drugs.

\section{Safety: How Much Is Enough?}

The question of how much safety data are sufficient for pre-symptomatic trials generated intense discussion. In general, if trial participants are considered healthy yet expected to take a drug for a long time, the safety bar is high, too high for most investigational AD drugs to pass at present. Reiman noted that safety is paramount in drug selection, saying "The 'go' decision for us is not whether we have efficacy in clinically affected patients, but whether we have safety." Typically, drugs tested in prevention trials are already approved by the FDA for other indications, but the group assembled in Phoenix appeared most interested in investigational treatments, primarily but not exclusively anti-amyloid agents. This more ambitious goal raised questions such as these: 
- Can Phase II safety data possibly be sufficient?

- If the trial is to start in 2011, will more than a handful of candidates even have more than Phase II safety?

- Will the FDA require carcinogenicity tests on any chosen drug?

- Regulatory agencies typically require at least 1,500 long-term exposure patients - how many treatments even have that?

- Who is more able to withstand any drug side effects anyway - a cognitively healthy younger person or a person diagnosed with $\mathrm{AD}$ ?

Some scientists felt that trial participants at highest risk of imminent $\mathrm{AD}$ required less stringent safety data than "regular" cognitively normal people. They argued that trial participants be involved in weighing their AD risk against possible drug risks. Some argued that people at risk for AD judge this issue differently than do their doctors and drug sponsors, who act to preclude legal consequences of drug side effects. Yet, there was no consensus on how to include people's rationalization of their personal risks. For example, Tariot asked, "If you ask 68-year-old ApoE4 homozygote people if they would accept the risk of vasogenic edema, some will say yes, others will say no. How do we handle that?" Others suggested that the less ambitious approach of enrolling participants whose cognition was already beginning to show signs of trouble would shift the risk equation toward treatment while also increasing the number of participants who will decline on placebo, i.e., make it easier to detect a clinical drug effect. This would not constitute pre-symptomatic treatment; rather, it would reflect the notion of conducting trials at what is sometimes called the early MCI (eMCI), or prodromal, stage of Alzheimer disease. Its downside, some noted, is that treatment may come too late to have its greatest possible effect.

At the end of the day, industry representatives agreed that they need to make haste in securing safety data. As one scientist put it, "We need to do now the appropriate safety and tolerability trials so we will be ready with that data by the time these prevention trials can be done."

\section{Efficacy: How Little Is Enough?}

Must a drug have shown efficacy in clinical trials of mild to moderate $\mathrm{AD}$ in order to be chosen for a pre-symptomatic trial? Reflecting recent trends in $\mathrm{AD}$ research, most attendees said no, though they added that they would feel more confident if the drug showed some signal, even a small one, in those trials. Even just for the purpose of setting the right dose, drug sponsors much prefer to have seen some sort of efficacy response to their drug. Everyone agreed on one bedrock requirement, however. To be selected, an API treatment must come with rigorous data showing that it has reached and engaged its intended molecular target, i.e., is biologically active in the brain. This can be done with biomarker readouts, but however it is accomplished, attendees insisted on solid target coverage data as an efficacy minimum if they are to take on the risk of presymptomatic research. This reflects, in part, lessons learned from the failure in Phase III trial of Flurizan. This drug was widely seen as a test of the amyloid hypothesis, but apparently went into Phase III without proof that enough of it reached and engaged its target, $\gamma$-secretase, in the human brain.

Beyond merely showing that the treatment engages its immediate molecular target, biomarker studies should show that it moves some of the most validated AD biomarkers in the expected direction, some scientists urged in Phoenix. One bold suggestion came up repeatedly: to inform the drug selection process, API scientists could request several candidate treatments and compare them side-by-side in short target engagement studies. These could use the biomarkers that most closely reflect the candidates' underlying medical hypotheses. Even if drug companies balked at direct comparison of their "assets" and ran such brief studies individually on their compounds, they could still generate the necessary data on bioavailability, exposure, and indeed dose, for a larger, longer API trial. Moreover, genetic high-risk populations may have more pathology than people with sporadic AD; hence, doses needed to move biomarkers must be set directly in them.

\section{Dose: Still a Black Box}

Industry scientists suggested that a pre-symptomatic drug trial should test two doses versus placebo - provided, that is, that the sample sizes receiving a particular dose are large enough to evaluate the drug with adequate statistical power. How to set those doses in this pre-symptomatic setting is still quite an open question. The API scientists had drafted a study protocol for discussion purposes. Reviewing it, the invited group made clear they would like to see much more information about how to set the dose. Finding a safe and effective dose is especially important if the trials are to take as long as currently proposed, i.e., five years. 
Yet discussion about dose, indeed about many protocol details, cannot get overly specific until a drug is at hand and its mechanism of action can be taken into consideration, scientists cautioned. Hence, this discussion largely awaits another day.

\section{PART 5: MAKING TRIALS WORK FOR PATIENT, SPONSOR, REGULATOR}

Exactly how to correctly fashion a pre-symptomatic trial appears to be in the eye of the beholder. This was the upshot of trial design discussions at an industry advisory meeting hosted last month by Eric Reiman, Pierre Tariot, and their colleagues from the Banner Alzheimer's Institute in Phoenix, Arizona, on their proposed API. The API scientists fielded ample advice, including this: "You will get a lot of informed opinions but no consensus on any details. You will have to make your decision and go with it." Here is a short list of questions that came up repeatedly:

- Enroll people regardless of their brain amyloid load, or only people who have amyloid? Each approach had its proponents. The API leaders at present are leaning toward including all who meet a combined genotype/age requirement and then analyzing the trial outcomes relative to how much amyloid deposition they had.

- What to designate as a primary endpoint? One or more biomarkers, followed by cognitive/clinical secondary endpoints? Or vice versa?

- Should the trial analyze biomarkers and clinical outcomes together or separately? Each statistic has regulatory consequences

- What would be the right clinical measure to determine if the drug has worked in people who were normal at the outset? This is the inherent conundrum of how one can prove prevention.

- What are these trials? Phase II? Phase III?

- Should API run pilot drug-biomarker studies and then hand the drug back to the sponsor for further development, or should the API reach for fullfledged, pivotal FDA registration trials?

- How do the API trials fit into a company's overall development plan for its drug?

Beyond that, there was a strong sense in the room that API should focus primarily on generating exciting science, not get overly concerned with obtaining regulatory support from the get-go. In other words, once data show a drug to have moved biomarkers in pre- symptomatic folks, regulators will be interested to find a path to approval. "If we can show that a given agent is effective in a prevention paradigm, then I am sure the regulatory agency can work with us," one industry scientist said.

Some trial design issues stood out for the broad consensus they drew. First among them, industry scientists said that in order to justify API trials internally, they would prefer a shorter design that delivers at least some interim data after two years. "We need some early successes to keep enthusiasm and funding going," one pharma representative said. The current trial design of five years, plus the time it takes to recruit and follow up, is too long, they said, if there is not a significant chance to learn something midway through. Drug sponsors do not want to treat for so long with a drug that might not work. Even the ADNI - a simpler proposition to begin with - offered short-term data to look at, which kept industry support going. Some attendees worried about the prospect of a fuzzy outcome at the end, where there is a hint but not proof of clinical efficacy. What to do then? Keep treating with study drug for another five years in the hope that the separation between placebo and study drug will be clearer? In this situation, a strong primary biomarker that closely reflects the medical hypothesis of the treatment can generate some confidence, these advisers said.

Attendees around the room voiced dissatisfaction with the standard parallel arm trial designs and the standard primary/secondary outcomes. Calling these "archaic," Lon Schneider from the University of Southern California in Los Angeles argued for more exploration of creative alternatives such as rolling starts and multivariate outcomes. These could even be customized to individual participants if they had undergone a period of intense observation prior to drug treatment, perhaps in a registry that uses social networking media, during which their biomarker levels were established or had even shown a trajectory of change. There is more than one advantage to that approach: "If you get trajectory of change on individuals, then the recruitment for a treatment trial will be a nanosecond," quipped Schneider (see expanded comment below). The DIAN is taking this approach of natural history preceding drug treatment. It could be broadened to other populations if large registries of cognitively normal study volunteers were built from which companies could then recruit participants for observational, dose-finding, or target engagement studies prior to moving on to formal treatment trials. This goal drew unanimous support.

Furthermore, industry scientists urged the API researchers to bolster the scientific data on which they 
base their conversion rates and subsequent power calculations for the ApoE trial. Frequently, the power seen in observational studies does not map onto subsequent trials, and trial planning needs to account for that. In terms of cognition, the API leaders heard advice to fine-tune their tests of whether trial participants were developing memory problems during the course of the trial to the drug at hand: i.e., use tests that stress hippocampal function if it is an anti-tau drug, or tests that probe attention if it is an anti-amyloid drug.

Finally, this point resonated throughout the day: Whatever specific design the API leaders settle on for their initial trials, science will learn a huge amount, and these trials will galvanize interest in prevention studies throughout the field. Pharma representatives from Europe asked to be included in the ApoE4 initiative as well.

Reiman concluded the day by saying: "Both the stakes and opportunity at hand could not be greater. If it is a choice between an incremental approach to this problem or a bold one, we will get further if we are bold. Evaluating drugs in the highest-risk populations is not only good for companies, but also good for the world, and we will move this process forward while trying to address each of the concerns and issues raised here today."

\section{REFERENCES}

[1] Reiman EM, Langbaum J, Tariot PN (2010) Alzheimer's Prevention Initiative: a proposal to evaluate presymptomatic treatments as quickly as possible. Biomarkers Med 4, 3-14.

[2] ADAPT Research Group, Martin BK, Szekely C, Brandt J, Piantadosi S, Breitner JC, Craft S, Evans D, Green R, Mullan M (2008) Cognitive function over time in the Alzheimer's Disease Anti-inflammatory Prevention Trial (ADAPT): results of a randomized, controlled trial of naproxen and celecoxib. Arch Neurol 65, 896-905.

[3] DeKosky ST, Williamson JD, Fitzpatrick AL, Kronmal RA, Ives DG, Saxton JA, Lopez OL, Burke G, Carlson MC, Fried LP, Kuller LH, Robbins JA, Tracy RP, Woolard NF, Dunn L, Snitz BE, Nahin RL, Furberg CD, Ginkgo Evaluation of Memory (GEM) Study Investigators (2008) Ginkgo biloba for prevention of dementia: a randomized controlled trial. JAMA 300, 2253-2262.

[4] Shumaker SA, Legault C, Rapp SR, Thal L, Wallace RB, Ockene JK, Hendrix SL, Jones BN, Assaf AR, Jackson RD, Kotchen JM, Wassertheil-Smoller S, Wactawski-Wende J, WHIMS Investigators (2003) Estrogen plus progestin and the incidence of dementia and mild cognitive impairment in postmenopausal women: the Women's Health Initiative Memory Study: a randomized controlled trial. JAMA 289, 2651-2662.

[5] Shumaker SA, Legault C, Kuller L, Rapp SR, Thal L, Lane DS, Fillit H, Stefanick ML, Hendrix SL, Lewis CE, Masaki K, Coker LH, Women's Health Initiative Memory Study (2004) Conjugated equine estrogens and incidence of probable dementia and mild cognitive impairment in postmenopausal women: Women's Health Initiative Memory Study. JAMA 291, 2947-2958.

[6] Alzheimer Research Forum (2009) News Brief: ASG's Strategic Report, Senate Testimony Freely Available, http://www. alzforum.org/new/detail.asp?id=2098.

[7] Lopera F, Ardilla A, Martínez A, Madrigal L, Arango-Viana JC, Lemere CA, Arango-Lasprilla JC, Hincapíe L, ArcosBurgos M, Ossa JE, Behrens IM, Norton J, Lendon C, Goate AM, Ruiz-Linares A, Rosselli M, Kosik KS (1997) Clinical features of early-onset Alzheimer disease in a large kindred with an E280A presenilin-1 mutation. JAMA 277, 793-799.

[8] Lemere CA, Lopera F, Kosik KS, Lendon CL, Ossa J, Saido TC, Yamaguchi H, Ruiz A, Martinez A, Madrigal L, Hincapie L, Arango JC, Anthony DC, Koo EH, Goate AM, Selkoe DJ, Arango JC (1996) The E280A presenilin 1 Alzheimer mutation produces increased A beta 42 deposition and severe cerebellar pathology. Nat Med 10, 1146-1150.

[9] Johnson KA, Lopera F, Jones K, Becker A, Sperling R, Hilson J, Londono J, Siegert I, Arcos M, Moreno S, Madrigal L, Ossa J, Pineda N, Ardila A, Roselli M, Albert MS, Kosik KS, Rios A (2001) Presenilin-1-associated abnormalities in regional cerebral perfusion. Neurology 56, 1545-1551.

[10] Pastor P, Roe CM, Villegas A, Bedoya G, Chakraverty S, García G, Tirado V, Norton J, Ríos S, Martínez M, Kosik KS, Lopera F, Goate AM (2003) Apolipoprotein Eepsilon4 modifies Alzheimer's disease onset in an E280A PS1 kindred. Ann Neurol 54, 163-169.

[11] Arango-Lasprilla JC, Cuetos F, Valencia C, Uribe C, Lopera F (2007) Cognitive changes in the preclinical phase of familial Alzheimer's disease. J Clin Exp Neuropsychol 29, 892-900.

[12] Kosik KS (1999) The fortune teller. The Sciences, July/August, 13-17; http://www.sbcfit.org/pdfs/fortune_teller.pdf.

[13] Reiman EM, Caselli RJ, Chen K, Alexander GE, Bandy D, Frost J (2001) Declining brain activity in cognitively normal apolipoprotein E epsilon 4 heterozygotes: A foundation for using positron emission tomography to efficiently test treatments to prevent Alzheimer's disease. Proc Natl Acad Sci U S A 98, 3334-3339.

[14] Reiman EM, Chen K, Alexander GE, Caselli RJ, Bandy D, Osborne D, Saunders AM, Hardy J (2005) Correlations between apolipoprotein E epsilon4 gene dose and brain-imaging measurements of regional hypometabolism. Proc Natl Acad Sci U S A 102, 8299-8302.

[15] Chen K, Reiman EM, Alexander GE, Caselli RJ, Gerkin R, Bandy D, Domb A, Osborne D, Fox N, Crum WR, Saunders AM, Hardy J (2007) Correlations between apolipoprotein E epsilon 4 gene dose and whole brain atrophy rates. Am J Psychiatry 164, 916-921.

[16] Reiman EM, Chen K, Liu X, Bandy D, Yu M, Lee W, Ayutyanont N, Keppler J, Reeder SA, Langbaum JB, Alexander GE, Klunk WE, Mathis CA, Price JC, Aizenstein HJ, Dekosky ST, Caselli RJ (2009) Fibrillar amyloid-beta burden in cognitively normal people at 3 levels of genetic risk for Alzheimer's disease. Proc Natl Acad Sci US A 106, 68206825 .

[17] Thambisetty M, Beason-Held L, An Y, Kraut MA, Resnick SM (2010) APOE epsilon4 genotype and longitudinal changes in cerebral blood flow in normal aging. Arch Neurol 67, 93-98.

[18] Caselli RJ, Dueck AC, Osborne D, Sabbagh MN, Connor DJ, Ahern GL, Baxter LC, Rapcsak SZ, Shi J, Woodruff BK, Locke DE, Snyder CH, Alexander GE, Rademakers R, Reiman EM (2009) Longitudinal modeling of age-related memory decline and the APOE epsilon4 effect. N Engl J Med 361, 255-263. 